\title{
Immune inflammatory modulation as a potential therapeutic strategy of stem cell therapy for ALS and neurodegenerative diseases
}

\author{
Seung Hyun Kim ${ }^{1,2, *}$, Ki-Wook $\mathrm{Oh}^{1,2}$, Hee Kyung Jin ${ }^{3,5}$ E Jae-Sung Bae \\ ${ }^{1}$ Department of Neurology, College of Medicine, Hanyang University, Seoul 04763, ${ }^{2}$ Cell Therapy Center for Neurological Disorders, \\ Hanyang University Hospital, Seoul 04763, ${ }^{3}$ Stem Cell Neuroplasticity Research Group, ${ }^{4}$ Department of Physiology, Cell and Matrix \\ Research Institute, School of Medicine, ${ }^{5}$ Department of Laboratory Animal Medicine, College of Veterinary Medicine, Kyungpook National \\ University, Daegu 41566, Korea
}

\begin{abstract}
With emerging evidence on the importance of non-cell autonomous toxicity in neurodegenerative diseases, therapeutic strategies targeting modulation of key immune cells. including microglia and Treg cells, have been designed for treatment of ALS and other neurodegenerative diseases. Strategy switching the patient's environment from a pro-inflammatory toxic to an anti-inflammatory, and neuroprotective condition, could be potential therapy for neurodegenerative diseases. Mesenchymal stem cells (MSCs) regulate innate and adaptive immune cells, through release of soluble factors such as TGF- $\beta$ and elevation of regulatory $\mathrm{T}$ cells (Tregs) and T helper-2 cells (Th2 cells), would play important roles, in the neuroprotective effect on motor neuronal cell death mechanisms in ALS. Single cycle of repeated intrathecal injections of BM-MSCs demonstrated a clinical benefit lasting at least 6 months, with safety, in ALS patients. Cytokine profiles of CSF provided evidence that BM-MSCs, have a role in switching from pro-inflammatory to anti-inflammatory conditions. Inverse correlation of TGF- $\beta 1$ and MCP-1 levels, could be a potential biomarker to responsiveness. Thus, additional cycles of BM-MSC treatment are required, to confirm long-term efficacy and safety. [BMB Reports: Perspective 2018; 51(11): 545-546]
\end{abstract}

*Corresponding author. E-mail: kimsh1@hanyang.ac.kr

https://doi.org/10.5483/BMBRep.2018.51.11.255

Received 23 October 2018

Keywords: ALS, Biomarker, BM-MSCs, Immune modulation, Neurodegenerative disease

Abbreviations: ALS, amyotrophic lateral sclerosis; BM-MSCs, bone marrow originated mesenchymal stem cells; CSF, cerebrospinal fluid

Perspective to: Oh KW 2018, Repeated Intrathecal Mesenchymal Stem Cells for Amyotrophic Lateral Sclerosis. Annals of Neurology. 2018;84(3):361-373. doi: 10.1002/ana.25302
Among recent breakthroughs in understanding pathogenic mechanisms of amyotrophic lateral sclerosis (ALS), important issues are summarized as follow: 1) Knowledge of novel causative genes expand concepts on ALS, towards motor neural network syndrome or multisystem proteinopathy. 2) The concept of non-cell autonomous effect on motor neuronal cell death mechanisms supports further study of immuneinflammatory modulation, as clinical therapeutic strategy for neurodegenerative diseases. Despite advances in understanding the connection of clinical heterogeneity, genetic and molecular mechanisms of motor neuronal death in ALS, previously conducted clinical trials have failed. Previous trials based on single molecular targets suggest the importance of integration, with multiple molecular targets in overall therapeutic strategy.

Mesenchymal stem cells (MSCs) therapy, is the desired approach for addressing this issue. MSCs exert diverse actions, such as stimulating intrinsic neurogenesis, releasing diverse neurotrophic factors, and modulating immune-inflammatory processes. Traditional stem cell therapy has been promoted as potential strategy for ALS, based on self-renewal and differentiation into multiple cell types, with the final goal of repairing or replacing injured cells. However, by simply replacing injured cells and differentiation into motor neurons, it would be difficult to expect new motor neurons to reproduce extensive connections from cortical neurons, to the brain-stem or spinal cord neuronal population. Too, new motor neurons, if integrated into a diseased network, may be subject to the same pathologic processes that resulted in demise of the original motor neurons (Appel SH et al (2016) Neurology 87, 348-349).

Thus, therapeutic strategy using stem cells should be switched towards neuroprotective goals, from the concept of neuronal replacement or reconstruction in neurodegenerative diseases. Repeated intrathecal MSCs therapy (Oh KW et al (2018) Ann Neurol 84, 361-373) empathized the importance of ethical issues and concept of enriched model for stratified medicine, as well as the significance of diverse biological markers, as a predicting tool for effectiveness and responsiveness of autologous MSCs treatment. Too, autologous

ISSN: 1976-670X (electronic edition)

Copyright (C) 2018 by the The Korean Society for Biochemistry and Molecular Biology

(c) This is an open-access article distributed under the terms of the Creative Commons Attribution Non-Commercial License (http://creativecommons.org/licenses/by-nc/4.0) which permits unrestricted non-commercial use, distribution, and reproduction in any medium, provided the original work is properly cited. 


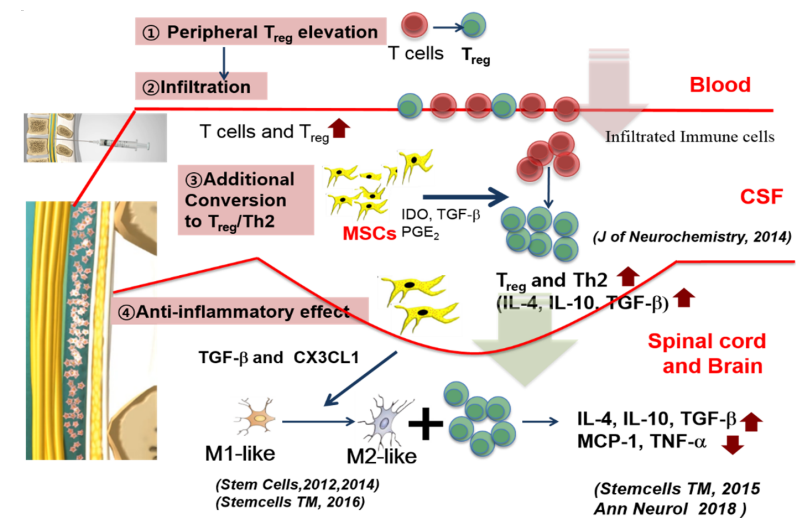

Diagram 1. Summary of plausible mechanisms of intrathecally injected BM-MSCs in ALS.

MSCs have multiple advantages in clinical practice, especially in relation to ethical concerns, lack of possible tumorigenesis, or graft rejections. MSCs regulate innate and adaptive immune cells, through release of soluble factors such as prostaglandin E2 (PGE2), indoleamine 2,3-dioxygenase (IDO), and TGF- $\beta$, thereby switching the patient's environment from a proinflammatory toxic to an anti-inflammatory, and neuroprotective condition. Recently, we reported that immunoregulatory mechanisms for MSCs, such as elevation of regulatory $\mathrm{T}$ cells (Tregs) and T helper-2 cells (Th2 cells), would play important roles in the neuroprotective effect on motor neuronal cell death mechanisms in ALS, similar secretion of neurotrophic factors crucial to the effectiveness of MSCs in ALS. In addition, MSCs can modulate functional properties of microglia via TGF- $\beta$ secretion, switching them from a classically activated phenotype to an inflammation-resolving phenotype. These modes of action mechanisms published in previous our data, are summarized in Diagram 1 and 2. These effects of MSCs could be important therapeutic strategy, to inhibit toxic neuroinflammatory processes in the symptomatic stage of ALS.

Data of our previous phase 1 clinical trial have shown that repeated intrathecal autologous bone marrow-derived mesenchymal stem cells (BM-MSCs) therapy is a promising therapeutic strategy, however, reliable biological markers predicting prognosis and/or delineating mechanisms on protecting cell death in ALS have not been fully described. Also, we reported that factors such as TGF- $\beta$, angiogenin (ANG), and vascular endothelial growth factor (VEGF), cytokines secreted by BM-MSCs, play crucial roles in the ALS patient's response to intrathecal autologous BM-MSCs injection. Based on known action mechanisms of MSCs and findings from in vivo transgenic mouse study, we hypothesized that repeated intrathecal BM-MSCs administration could be a valuable therapeutic strategy for ALS. After completion of this first randomized, controlled trials designed to evaluate safety

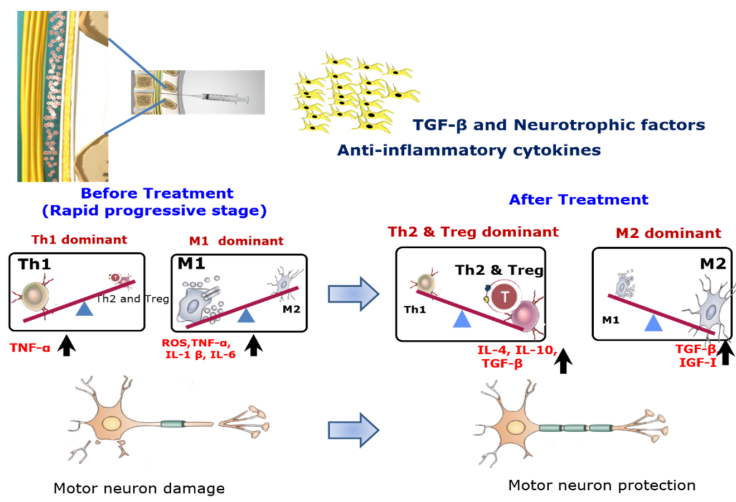

Diagram 2. Hypothesized mechanisms of repeated intrathecal autologous MSCs therapy for ALS when focused on changes from pre-treated state to post-treated one of key immune-inflammatory cells.

and efficacy of repeated intrathecal BM-MSC therapy in ALS patients, post-hoc analysis of candidate biological markers, and genetic and clinical features, have been conducted. Repeated intrathecal injections of MSCs demonstrated a possible clinical benefit, on functional decline with safety in ALS patients. Cytokine profiles of CSF provided evidence that BM-MSCs have a role, in switching from pro-inflammatory to anti-inflammatory conditions. The inverse correlation of TGF- $\beta 1$ and MCP- 1 level could be potential biomarker to responsiveness.

This study was enriched in that it considered clinical findings, but also genetic aspects, by excluding known common mutations that may be seen even in sporadic ALS. Too, relevance of CSF biomarkers was evaluated, for depth of understanding the underlying immunomodulatory mechanism of BM-MSCs.

Post-hoc survival analysis, did not show significant difference between the two groups. Despite the positive effect on ALSFRS-R lasting at least 6 months, lack of long-term survival benefit may be associated with the number of treatments of MSC with two limited injections in this trial protocol. The potential therapeutic effect of BM-MSCs would not be longlasting, because BM-MSCs gradually disappear over time in CSF. Considering the immune modulatory effect of BM-MSC treatment using less-invasive procedures, serial additional BM-MSC treatments after 6 months may improve long-term efficacy.

\section{ACKNOWLEDGEMENTS}

This study was supported by the National Research Foundation (NRF) of Korea funded by the Korea Ministry of Science (2018M3C7A1056512). And also supported by the Korea Health Technology R\&D Project through the Korea Health Industry, Development Institute (KHIDI), funded by the Ministry of Health \& Welfare, Republic of Korea (HI16C2131). 